\title{
THE DISCRETE MEAN SQUARE OF THE DIRICHLET $L$-FUNCTION AT NONTRIVIAL ZEROS OF ANOTHER DIRICHLET L-FUNCTION
}

\author{
RAMŪNAS GARUNKŠTIS* and JUSTAS KALPOKAS ${ }^{\dagger}$ \\ Faculty of Mathematics and Informatics, Vilnius University \\ Naugarduko 24, 03225 Vilnius, Lithuania \\ *ramunas.garunkstis@mif.vu.lt \\ †justas.kalpokas@mif.vu.lt
}

Received 25 June 2012

Accepted 6 December 2012

Published 25 March 2013

\begin{abstract}
We consider the sum of squared absolute values of the Dirichlet $L$-function taken at the nontrivial zeros of another Dirichlet $L$-function.

Keywords: Dirichlet $L$-functions; distribution of zeros; common zeros of Dirichlet $L$ functions; second moment.
\end{abstract}

Mathematics Subject Classification 2010: 11M06, 11M26

\section{Introduction}

Let $s=\sigma+i t$ denote a complex variable. The Dirichlet $L$-function is defined by

$$
L(s, \chi)=\sum_{n=1}^{\infty} \frac{\chi(n)}{n^{s}} \quad(\sigma>1),
$$

where $\chi(n)$ is a Dirichlet character modulo $q$. For $\chi \bmod 1$ we get the Riemann zeta-function $L(s, \chi)=\zeta(s)$. The Generalized Riemann Hypothesis (GRH) states that inside the critical strip $0<\sigma<1$ every Dirichlet $L$-function has zeros only on the critical line $\sigma=\frac{1}{2}$. Zeros in the critical strip are called nontrivial and we denote them by $\rho_{\chi}=\beta_{\chi}+i \gamma_{\chi}$. A Dirichlet character $\chi \bmod q$ is said to be primitive if it is not induced by any other character of modulus strictly less than $q$. The unique principal character modulo $q$ is denoted by $\chi_{0}$. The character $\chi_{0} \bmod 1$ is the only principal and primitive character. For a Dirichlet character $\chi \bmod q$ the associated Gauss sum is defined by

$$
G(n, \chi)=\sum_{a=1}^{q} \chi(a) \exp \left(2 \pi i \frac{a n}{q}\right) .
$$


If $n=1$ we denote $\tau(\chi)=G(1, \chi)$. For a primitive character $\chi \bmod q$ we have $|\tau(\chi)|=\sqrt{q}$ and for the principal character $\chi_{0}$ we have $\tau\left(\chi_{0}\right)=\mu(q)$, where $\mu(q)$ is the Möbius function.

In this paper $T$ always tends to plus infinity, $\varepsilon$ is any positive real number, and $p$ denotes a prime number. Let $\phi$ denote the Euler totient function and let

$$
D(Q)=2 \sum_{p \mid Q} \frac{\log p}{p-1}+2 \gamma,
$$

where $\gamma$ is the Euler constant. Further let $\delta(q, Q)=1$, if $q \mid Q, \delta(q, Q)=0$ otherwise. We shall prove the following result.

Theorem 1.1. Let $A$ and $B$ be positive constants. Let $\psi \bmod Q$ and $\chi \bmod q$ be primitive Dirichlet characters and $\chi \neq \psi$. Then, uniformly for $Q \ll \log ^{A} T$ and $q \ll \log ^{B} T$, we have

$$
\begin{aligned}
\sum_{0<\gamma_{\chi} \leq T} L\left(\rho_{\chi}, \psi\right) L\left(1-\rho_{\chi}, \bar{\psi}\right)= & \frac{\phi(Q)}{Q} \frac{T}{2 \pi} \log ^{2} \frac{T}{2 \pi e}+a_{1} \frac{T}{2 \pi} \log \frac{T}{2 \pi e}+a_{2} \frac{T}{2 \pi} \\
& +O\left(T^{\left.1-\frac{c}{\log ^{\frac{3}{4}+\varepsilon} T}\right),}\right.
\end{aligned}
$$

where

$$
a_{1}=\frac{\phi(Q)}{Q}\left(\log (Q q)+D(Q)+2 \Re \frac{L^{\prime}}{L}(1, \chi \bar{\psi})\right)
$$

and

$$
\begin{aligned}
a_{2}= & \frac{\phi(Q)}{Q}\left[\log Q \log q+D(Q) \log q+1+2(\log Q+D(Q)) \Re \frac{L^{\prime}}{L}(1, \chi \bar{\psi})\right. \\
& \left.+2 \Re\left(\frac{L^{\prime \prime}}{L}-\left(\frac{L^{\prime}}{L}\right)^{2}\right)(1, \chi \bar{\psi})\right]-2 \delta(q, Q)(\chi \bar{\psi})(-1) \\
& \times \Re\left(\frac{\tau\left(\chi \psi_{0}\right) \tau(\bar{\psi})}{\phi(Q)} L^{2}(1, \chi \bar{\psi})\right) .
\end{aligned}
$$

If we assume GRH then the left-hand side of the last equality can be replaced by $\sum_{0<\gamma_{\chi} \leq T}\left|L\left(\frac{1}{2}+i \gamma_{\chi}, \psi\right)\right|^{2}$ and the error term can be replaced by $O\left(q^{1+\varepsilon} Q^{\varepsilon} T^{\frac{1}{2}+\varepsilon}+\right.$ $\left.q Q^{\frac{9}{2}+\varepsilon} T^{\varepsilon}+(Q T)^{\frac{1}{2}+\varepsilon}\right)$ uniformly for all $Q$ and $q$.

From Theorem 1.1 we immediately deduce the following corollary.

Corollary 1.2. Let $\psi \bmod Q$ and $\chi \bmod q$ be primitive Dirichlet characters and $\chi \neq \psi$. Assume that all nontrivial zeros of $L(s, \chi)$ lie on the critical line. Then the sequence $\left(L\left(\rho_{\chi}, \psi\right)\right)$, where $\rho_{\chi}$ runs over all nontrivial zeros of $L(s, \chi)$, is unbounded.

Theorem 1.1 is related to the following theorem proved in [11]. 
Theorem 1.3. Let $A$ and $B$ be positive constants. Let $\psi \bmod Q$ and $\chi \bmod q$ be primitive Dirichlet characters and $\chi \neq \psi$. Then, uniformly for $Q \ll \log ^{A} T$ and $q \ll \log ^{B} T$, we have

$$
\begin{aligned}
\sum_{0<\gamma_{\chi} \leq T} L\left(\rho_{\chi}, \psi\right)= & \frac{T}{2 \pi} \log \frac{T q}{2 \pi e}-\delta(q, Q) L(1, \chi \bar{\psi}) \psi(-1) \tau(\psi) \frac{\tau\left(\bar{\chi} \psi_{0}\right)}{\phi(Q)} \frac{T}{2 \pi} \\
& +\frac{L^{\prime}}{L}(1, \psi \bar{\chi}) \frac{T}{2 \pi}+O\left(T \exp \left(-c \log ^{\frac{1}{4}-\varepsilon} T\right)\right),
\end{aligned}
$$

where $c$ is a positive absolute constant.

Under GRH the error term can be replaced by $O\left((T Q)^{1 / 2+\varepsilon} q^{\varepsilon}\right)$, which is valid uniformly for $q, Q \ll T^{1-\varepsilon}$.

Assuming GRH for $L(s, \chi)$ Theorem 1.3 was proved by Fujii [8] with the error term $O\left(T \exp \left(-c \log ^{\frac{1}{2}} T\right)\right)$. Later Steuding [19] proved Theorem 1.3 unconditionally with the error term $O(T)$. See also [14], where the case $Q=1$ and $q \leq \exp \left(c \sqrt{\log \frac{T}{2 \pi}}\right), c$ being an appropriate positive constant, was considered.

Theorems 1.1 and 1.3 lead to the next corollary.

Corollary 1.4. Let $\psi \bmod Q$ and $\chi \bmod q$ be primitive Dirichlet characters and $\chi \neq \psi$. Assume that all nontrivial zeros of $L(s, \chi)$ lie on the critical line. Let $A$ be any positive real number. Then, uniformly for $q \ll(\log T)^{A}$ and $Q \ll(\log T)^{2-\varepsilon}$, we have

$$
\sum_{\substack{0<\gamma_{\chi} \leq T \\ L\left(1 / 2+\gamma_{\chi}, \psi\right) \neq 0}} 1 \gg \frac{Q}{\phi(Q)} T .
$$

The above results give certain information concerning the relationship of zeros of two Dirichlet $L$-functions. The conjecture is that different primitive Dirichlet $L$-functions have no common nontrivial zeros (see $[9$, Conjecture $3 ; 16]$ ). Towards this hypothesis Fujii [7] unconditionally obtained, that if $\chi_{1}$ and $\chi_{2}$ are different primitive characters to the same modulus $q$, then the positive proportion of zeros of $L\left(s, \chi_{1}\right)$ and $L\left(s, \chi_{2}\right)$ are non-coincident. Here a zero $\rho$ is called a coincident zero of $L\left(s, \chi_{1}\right)$ and $L\left(s, \chi_{2}\right)$ if $L\left(\rho, \chi_{1}\right)=L\left(\rho, \chi_{2}\right)=0$ with the same multiplicity. Assuming the Riemann Hypothesis for $\zeta(s)$ Conrey, Ghosh, and Gonek $[2,3]$ proved that at most two-thirds of zeros of $\zeta(s)$ are also zeros of $L(s, \chi)$, where $\chi$ is a nonprinciple character. Conrey, Ghosh, and Gonek $[2,3]$ also note that their method allows to show (under GRH) that any two Dirichlet $L$-functions with inequivalent characters have at most two-thirds of zeros in common. For related results see also [18].

In the following section we will recall some basic facts about the analytic properties of Dirichlet $L$-functions and will give several lemmas. In Secs. 3 and 4 we will prove Theorem 1.1 and Corollary 1.4, respectively. 


\section{Auxiliary Results}

First we recall several facts about Dirichlet $L$-functions. Dirichlet $L$-function to a primitive character $\psi \bmod Q$ satisfies the functional equation (see [1, Theorem 12.11])

$$
L(1-s, \psi)=\tau(\psi) \frac{1}{Q}\left(\frac{Q}{2 \pi}\right)^{s} \Gamma(s)\left(\exp \left(-\frac{\pi i s}{2}\right)+\psi(-1) \exp \left(\frac{\pi i s}{2}\right)\right) L(s, \bar{\psi}) .
$$

Thus theorem from [13] and an application of the Phragmén-Lindelöf principle yield the estimates

$$
\begin{aligned}
& L(s, \psi) \ll|Q T|^{\frac{3}{16}+\varepsilon} \quad \text { for } \frac{1}{2} \leq \sigma \leq 1+\frac{1}{\log (Q T)},|t| \geq 1, \\
& L(s, \psi) \ll|Q T|^{\frac{1}{2}+\varepsilon} \quad \text { for }-\frac{1}{\log (Q T)} \leq \sigma<\frac{1}{2},|t| \geq 1
\end{aligned}
$$

uniformly in $|t| \ll T$. Under GRH the bound (2.2) can be replaced by

$$
L(s, \psi) \ll|Q T|^{\varepsilon} \quad \text { for } \frac{1}{2} \leq \sigma \leq 1+\frac{1}{\log (Q T)},|t| \geq 1
$$

uniformly in $|t| \ll T$. The bound (2.4) can be obtained similarly as in [20, Theorem 13.5], see also [10, Theorem 4].

We rewrite the functional equation (2.1) as

$$
L(1-s, \psi)=\Delta(1-s, \psi) L(s, \bar{\psi}),
$$

where $\Delta(s, \psi)$ is a meromorphic function with only real zeros and poles satisfying the functional equation

$$
\Delta(s, \psi) \Delta(1-s, \bar{\psi})=1 .
$$

Stirling's formula gives, for $t>1$,

$$
\Delta(\sigma+i t, \psi)=\left(\frac{t Q}{2 \pi}\right)^{\sigma-\frac{1}{2}-i t} \exp \left(i\left(t+\frac{\pi}{4}\right)\right)\left(1+O\left(\frac{1}{t}\right)\right)
$$

and

$$
\frac{\Delta^{\prime}}{\Delta}(s, \psi)=\frac{\Delta^{\prime}}{\Delta}(1-s, \bar{\psi})=-\log \frac{t Q}{2 \pi}+O\left(\frac{1}{t}\right) .
$$

Logarithmic differentiation of (2.5) leads to

$$
\frac{L^{\prime}}{L}(1-s, \chi)=\frac{\Delta^{\prime}}{\Delta}(1-s, \chi)-\frac{L^{\prime}}{L}(s, \bar{\chi}) .
$$

For the logarithmic derivative we have the partial fraction decomposition (see [17, Chap. 7, Theorem 4.1])

$$
\frac{L^{\prime}}{L}(s, \chi)=\sum_{\left|t-\gamma_{\chi}\right| \leq 1} \frac{1}{s-\rho_{\chi}}+O(\log (q(|t|+2))) \quad \text { for }-1 \leq \sigma \leq 2, t \geq 1 .
$$


For $q \geq 1, \chi \bmod q$ and $t \geq 0$ we have (see [17, Chap. 7, Theorem 3.3])

$$
\begin{aligned}
N_{\chi}(t+1)-N_{\chi}(t) & :=\#\left\{\rho_{\chi}=\beta_{\chi}+i \gamma_{\chi}: t<\gamma_{\chi} \leq t+1\right\} \\
& \ll \log (q(t+2)) .
\end{aligned}
$$

Thus the zeros $\rho_{\chi}$ cannot lie too dense: for any given $t_{0} \geq 2$ there exists a $t=t(\chi)$, $t \in\left(t_{0}, t_{0}+1\right]$, such that

$$
\min _{\gamma_{\chi}}\left|t-\gamma_{\chi}\right| \gg \frac{1}{\log (q t)} .
$$

In view of the expression (2.10) we get, for $t$ satisfying (2.12),

$$
\frac{L^{\prime}}{L}(\sigma+i t, \chi) \ll \log ^{2}(q t) \quad \text { for }-1 \leq \sigma \leq 2, \quad t \geq 2 .
$$

In the proof of Theorem 1.1 the following modified Gonek lemma (cf. [12, Lemma 5] and [5, Lemma 2]) will be important.

Lemma 2.1. Assume that $\sum_{n=1}^{\infty} \frac{a(n)}{n^{s}}$ converges for $\sigma>1$ and $a(n)=O\left(n^{\varepsilon}\right)$. Let $a=1+\frac{1}{\log (Q T)}$. Then

$$
\begin{aligned}
\frac{1}{2 \pi i} \int_{a+i}^{a+i T}\left(\frac{m}{2 \pi}\right)^{s} \Gamma(s) \exp \left(\delta \frac{\pi i s}{2}\right) \sum_{n=1}^{\infty} \frac{a(n)}{n^{s}} d s \\
\quad= \begin{cases}\sum_{n \leq \frac{T m}{2 \pi}} a(n) \exp \left(-2 \pi i \frac{n}{m}\right)+O\left(m^{a} T^{1 / 2+\varepsilon}\right) & \text { if } \delta=-1, \\
O\left(m^{a}\right) & \text { if } \delta=+1 .\end{cases}
\end{aligned}
$$

Proof. This is Lemma 2 in [11].

The next two technical lemmas will be useful in the proof of Theorem 1.1.

Lemma 2.2. Let $\chi \bmod q$ and $\psi \bmod Q$ be primitive Dirichlet characters and $\chi \neq$ $\psi$ with $Q \ll \log ^{A} T$ and $q \ll \log ^{B} T$, where $A$ and $B$ are positive constants. We have

$$
\begin{aligned}
& \sum_{m n l \leq \frac{T Q}{2 \pi}} \Lambda(m) \chi(m) \psi(n) \psi(l) \exp \left(-2 \pi i \frac{m n l}{Q}\right) \\
& =-\psi(-1) \tau(\psi) \frac{\phi(Q)}{Q} \frac{L^{\prime}}{L}(1, \chi \bar{\psi}) \frac{T}{2 \pi}\left(\log \frac{T Q}{2 \pi e}+2 \gamma+2 \sum_{p \mid Q} \frac{\log p}{p-1}\right. \\
& \left.\quad+\left(\frac{L^{\prime \prime}}{L^{\prime}}-\frac{L^{\prime}}{L}\right)(1, \chi \bar{\psi})\right)+\delta(q, Q) \chi(-1) \tau\left(\chi \psi_{0}\right) \frac{1}{\phi(Q)} \frac{T Q}{2 \pi} L^{2}(1, \chi \bar{\psi}) \\
& \quad+O\left(T^{\left.1-\frac{c}{\log ^{\frac{3}{4}+\varepsilon} T}\right) .}\right.
\end{aligned}
$$


Under GRH the error term can be replaced by

$$
O\left(q Q(T Q)^{\frac{1}{2}+\varepsilon} \log ^{2} q+q Q^{5} \log (Q T)\right)
$$

uniformly for all $Q$ and $q$.

Proof. By the orthogonality of Dirichlet characters the left-hand side of the formula in Lemma 2.2 can be written in the following way:

$$
\begin{aligned}
& \sum_{m n l \leq \frac{T Q}{2 \pi}} \Lambda(m) \chi(m) \psi(n) \psi(l) \exp \left(-2 \pi i \frac{m n l}{Q}\right) \\
& =\sum_{a=1}^{[q, Q]} \chi(a) \sum_{b=1}^{Q} \psi(b) \sum_{c=1}^{Q} \psi(c) \exp \left(-2 \pi i \frac{a b c}{Q}\right) \sum_{\substack{m n l \leq \frac{T Q}{2 \pi} \\
m \equiv a \bmod [q, Q] \\
n \equiv b \bmod Q \\
l \equiv c \bmod Q}} \Lambda(m) \\
& =\frac{1}{\phi^{2}(Q)} \sum_{\substack{\omega^{\prime} \bmod Q \\
\omega^{\prime \prime} \bmod Q}} \sum_{a=1}^{[q, Q]} \chi(a) \sum_{b=1}^{Q} \overline{\omega^{\prime}}(b) \psi(b) \sum_{c=1}^{Q} \frac{\omega^{\prime \prime}(c) \psi(c) \exp \left(-2 \pi i \frac{a b c}{Q}\right)}{\omega^{\prime}}(2 . \\
& \quad \sum_{\substack{m n l \leq \frac{T Q}{2 \pi} \\
m \equiv a \bmod [q, Q]}} \Lambda(m) \omega^{\prime}(n) \omega^{\prime \prime}(l)=S_{1}+S_{2}+S_{3}+S_{4},
\end{aligned}
$$

where $S_{j}, j=1,2,3,4$ correspond to the following cases:

- $S_{1}: \omega^{\prime}=\omega_{0}^{\prime}$ and $\omega^{\prime \prime}=\omega_{0}^{\prime \prime}$,

- $S_{2}: \omega^{\prime}=\omega_{0}^{\prime}$ and $\omega^{\prime \prime} \neq \omega_{0}^{\prime \prime}$,

- $S_{3}: \omega^{\prime} \neq \omega_{0}^{\prime}$ and $\omega^{\prime \prime}=\omega_{0}^{\prime \prime}$,

- $S_{4}: \omega^{\prime} \neq \omega_{0}^{\prime}$ and $\omega^{\prime \prime} \neq \omega_{0}^{\prime \prime}$,

here $\omega_{0}^{\prime}=\omega_{0}^{\prime \prime}$ is the principal character modulo $Q$.

First we consider $S_{1}$. Since $\psi$ is a primitive Dirichlet character, the Gauss sum is separable, i.e. $G(-a b, \psi)=\bar{\psi}(-a b) \tau(\psi)$ (see [6, Sec. 9]). By this and by the orthogonality of Dirichlet characters we have

$$
\begin{aligned}
S_{1}= & \psi(-1) \tau(\psi) \frac{1}{\phi(Q)} \sum_{a=1}^{[q, Q]} \chi(a) \bar{\psi}(a) \sum_{\substack{m n l \leq \frac{T Q}{2 \pi} \\
m \equiv a \bmod [q, Q]}} \Lambda(m) \omega_{0}^{\prime}(n) \omega_{0}^{\prime \prime}(l) \\
= & \psi(-1) \tau(\psi) \frac{1}{\phi([q, Q])} \frac{1}{\phi(Q)} \sum_{\eta \bmod [q, Q]} \sum_{a=1}^{[q, Q]} \bar{\eta}(a) \chi(a) \bar{\psi}(a) \\
& \times \sum_{m n l \leq \frac{T Q}{2 \pi}} \Lambda(m) \eta(m) \omega_{0}^{\prime}(n) \omega_{0}^{\prime \prime}(l) .
\end{aligned}
$$


In the last formula the sum over $a$ is not equal to zero if and only if $\eta=\chi \bar{\psi}$. Thus

$$
S_{1}=\psi(-1) \tau(\psi) \frac{1}{\phi(Q)} \sum_{m n l \leq \frac{T Q}{2 \pi}} \Lambda(m)(\chi \bar{\psi})(m) \omega_{0}^{\prime}(n) \omega_{0}^{\prime \prime}(l) .
$$

Second we consider $S_{2}$. In formula (2.14) interchanging the summation (over $b$ and $c$ ) and using the equality $G(-a c, \psi)=\bar{\psi}(-a c) \tau(\psi)$ we obtain

$$
\begin{aligned}
S_{2}= & \frac{1}{\phi^{2}(Q)} \sum_{\substack{\omega^{\prime \prime m o d}, Q \\
\omega^{\prime \prime} \neq \omega_{0}^{\prime \prime}}} \sum_{a=1}^{[q, Q]} \chi(a) \sum_{c=1}^{Q} \overline{\omega^{\prime \prime}}(c) \psi(c) \sum_{b=1}^{Q} \psi(b) \exp \left(-2 \pi i \frac{a b c}{Q}\right) \\
& \times \sum_{\substack{m n l \leq \frac{T Q}{2 \pi} \\
m \equiv a \bmod [q, Q]}} \Lambda(m) \omega_{0}^{\prime}(n) \omega^{\prime \prime}(l) \\
= & \psi(-1) \tau(\psi) \frac{1}{\phi^{2}(Q)} \sum_{\omega^{\prime \prime} \bmod Q} \sum_{\omega^{\prime \prime} \neq \omega_{0}^{\prime \prime}}^{[q, Q]} \chi(a) \bar{\psi}(a) \sum_{c=1}^{Q} \overline{\omega^{\prime \prime}}(c) \\
& \times \sum_{\substack{m n l \leq \frac{T Q}{2 \pi} \\
m \equiv a \bmod [q, Q]}} \Lambda(m) \omega_{0}^{\prime}(n) \omega^{\prime \prime}(l) .
\end{aligned}
$$

Hence $S_{2}=0$, since the sum over $c$ is equal to zero.

In the same way we obtain $S_{3}=0$.

Finally we consider $S_{4}$. First we note that, for $\omega^{\prime} \neq \omega_{0}^{\prime}, \omega^{\prime \prime} \neq \omega_{0}^{\prime \prime}$ and $(a,[q, Q])>1$,

$$
\begin{aligned}
\sum_{\substack{m \leq \frac{T Q}{2 \pi} \\
m \equiv a \bmod [q, Q]}} \Lambda(m) \sum_{n l \leq \frac{T Q}{2 \pi m}} \omega^{\prime}(n) \omega^{\prime \prime}(l) \mid & \leq \phi^{2}(Q) \sum_{\substack{m \leq \frac{T Q}{2 \pi} \\
m \equiv a \bmod [q, Q]}} \Lambda(m) \\
& =O\left(\phi^{2}(Q) \log (T Q)\right) .
\end{aligned}
$$

This yields

$$
\begin{aligned}
S_{4}= & \frac{1}{\phi^{2}(Q)} \sum_{\substack{\omega^{\prime} \bmod Q \\
\omega^{\prime} \neq \omega_{0}^{\prime}}} \sum_{\substack{\omega^{\prime \prime} \bmod Q \\
\omega^{\prime \prime} \neq \omega_{0}^{\prime \prime}}} \sum_{a=1}^{[q, Q]} \chi(a) \sum_{b=1}^{Q} \overline{\omega^{\prime}}(b) \psi(b) \\
& \times \sum_{c=1}^{Q} \overline{\omega^{\prime \prime}}(c) \psi(c) \exp \left(-2 \pi i \frac{a b c}{Q}\right) \\
& \times \sum_{\substack{m n l \leq \frac{T Q}{2 \pi} \\
m \equiv a \bmod [q, Q] \\
(a,[q, Q])=1}} \Lambda(m) \omega^{\prime}(n) \omega^{\prime \prime}(l)+O\left(\phi^{4}(Q) \phi([q, Q]) \log (T Q)\right) .
\end{aligned}
$$


If $(a b, Q)=1$, then $G\left(-a b, \overline{\omega^{\prime \prime}} \psi\right)=\left(\omega^{\prime \prime} \bar{\psi}\right)(-a b) \tau\left(\overline{\omega^{\prime \prime}} \psi\right)$ (see [6, Sec. 9]). Then by the orthogonality of Dirichlet characters we have

$$
\begin{aligned}
S_{4}= & \frac{1}{\phi([q, Q])} \frac{1}{\phi^{2}(Q)} \sum_{\eta \bmod [q, Q]} \sum_{\substack{\omega^{\prime} \bmod Q \\
\omega^{\prime} \neq \omega_{0}^{\prime \prime}}} \sum_{\substack{\omega^{\prime \prime} \bmod Q \\
\omega^{\prime \prime} \neq \omega_{0}^{\prime \prime}}}\left(\omega^{\prime \prime} \bar{\psi}\right)(-1) \tau\left(\overline{\omega^{\prime \prime}} \psi\right) \\
& \times \sum_{a=1}^{[q, Q]} \bar{\eta}(a) \chi(a) \omega^{\prime \prime}(a) \bar{\psi}(a) \sum_{b=1}^{Q} \overline{\omega^{\prime}}(b) \omega^{\prime \prime}(b) \sum_{m n l \leq \frac{T Q}{2 \pi}} \Lambda(m) \eta(m) \omega^{\prime}(n) \omega^{\prime \prime}(l) \\
& +O\left(\phi^{4}(Q) \phi([q, Q]) \log (T Q)\right) .
\end{aligned}
$$

In the last formula the sum over $b$ does not vanish if and only if $\omega^{\prime}=\omega^{\prime \prime}$. Further we write $S_{4}=T_{1}+T_{2}$, where $T_{1}$ and $T_{2}$ correspond to the following cases:

- $T_{1}: \eta=\eta_{0}$,

- $T_{2}: \eta \neq \eta_{0}$.

Let $\delta(q, Q)=1$ if $q \mid Q$ and $\delta(q, Q)=0$ otherwise. Then

$$
\begin{aligned}
T_{1}= & \frac{1}{\phi([q, Q])} \frac{1}{\phi(Q)} \sum_{\substack{\omega^{\prime} \bmod Q \\
\omega^{\prime} \neq \omega_{0}^{\prime}}}\left(\omega^{\prime} \bar{\psi}\right)(-1) \tau\left(\overline{\omega^{\prime}} \psi\right) \sum_{a=1}^{[q, Q]} \eta_{0}(a) \chi(a) \omega^{\prime}(a) \bar{\psi}(a) \\
& \times \sum_{m n l \leq \frac{T Q}{2 \pi}} \Lambda(m) \eta_{0}(m) \omega^{\prime}(n) \omega^{\prime \prime}(l) \\
= & \delta(q, Q)\left(\chi \psi_{0}\right)(-1) \tau\left(\chi \psi_{0}\right) \frac{1}{\phi(Q)} \\
& \times \sum_{m n l \leq \frac{T Q}{2 \pi}} \Lambda(m) \eta_{0}(m)(\bar{\chi} \psi)(n)(\bar{\chi} \psi)(l),
\end{aligned}
$$

since the sum over $a$ does not vanish if and only if $\omega^{\prime}=\bar{\chi} \psi$; and this equality is true if and only if $q \mid Q$. By definition of $T_{2}$ we have

$$
\begin{aligned}
T_{2}= & \frac{1}{\phi([q, Q])} \frac{1}{\phi(Q)} \sum_{\substack{\eta \bmod [q, Q] \\
\eta \neq \eta_{0}}} \sum_{\substack{\omega^{\prime} \bmod Q \\
\omega^{\prime} \neq \omega_{0}^{\prime}}}\left(\omega^{\prime} \bar{\psi}\right)(-1) \tau\left(\overline{\omega^{\prime}} \psi\right) \\
& \times \sum_{a=1}^{[q, Q]} \bar{\eta}(a) \chi(a) \omega^{\prime}(a) \bar{\psi}(a) \sum_{m n l \leq \frac{T Q}{2 \pi}} \Lambda(m) \eta(m) \omega^{\prime}(n) \omega^{\prime \prime}(l),
\end{aligned}
$$

where $\omega^{\prime \prime}$ is a non-principal character $\bmod Q$.

In view of the above we can write

$$
\begin{aligned}
& \sum_{m n l \leq \frac{T Q}{2 \pi}} \Lambda(m) \chi(m) \psi(n) \psi(l) \exp \left(-2 \pi i \frac{m n l}{Q}\right) \\
& =S_{1}+T_{1}+T_{2} O\left(\phi^{4}(Q) \phi([q, Q]) \log (T Q)\right),
\end{aligned}
$$


where $S_{1}, T_{1}$, and $T_{2}$ are given by formulae (2.15), (2.16), and (2.17). We will see that sums $S_{1}$ and $T_{1}$ produce the main term, and the sum $T_{2}$ contributes to the error term.

Next we consider $S_{1}$. Note that by conditions of the theorem the character $\chi \bar{\psi}$ is a non-principal character. By Perron's formula (see [20, Lemma 3.12])

$$
\begin{aligned}
-\frac{\phi(Q)}{\psi(-1) \tau(\psi)} S_{1}= & -\sum_{m n l \leq \frac{T Q}{2 \pi}} \Lambda(m)(\chi \bar{\psi})(m) \omega_{0}^{\prime}(n) \omega_{0}^{\prime \prime}(l) \\
= & \frac{1}{2 \pi i} \int_{a-i U}^{a+i U} \frac{L^{\prime}}{L}(s, \chi \bar{\psi}) L\left(s, \omega_{0}^{\prime}\right) L\left(s, \omega_{0}^{\prime \prime}\right)\left(\frac{T Q}{2 \pi}\right)^{s} \frac{d s}{s} \\
& +O\left(\frac{T Q \log ^{2}(T Q)}{U}\right),
\end{aligned}
$$

where $a=1+1 / \log (T Q)$.

In [17, Chap. 8, Theorem 6.2] considering $q \ll \log ^{B} T$ with $B$ being any positive constant we find that

$$
L(s, \chi) \neq 0 \quad \text { for } \sigma>1-\frac{c}{\log ^{\frac{3}{4}+\varepsilon} T},
$$

where $c$ is an absolute positive constant. With regard to this zero-free region for $L(s, \chi)$ let $b_{1}=1-c / \log ^{\frac{3}{4}+\varepsilon} T$. Shifting the line of integration and noting that $L\left(s, \omega_{0}^{\prime}\right)=L\left(s, \omega_{0}^{\prime \prime}\right)$ we get

$$
\begin{aligned}
& -\sum_{m n l \leq \frac{T Q}{2 \pi}} \Lambda(m)(\chi \bar{\psi})(m) \omega_{0}^{\prime}(n) \omega_{0}^{\prime \prime}(l) \\
& =\operatorname{Res}_{s=1} \frac{L^{\prime}}{L}(s, \chi \bar{\psi}) L^{2}\left(s, \omega_{0}^{\prime}\right)\left(\frac{T Q}{2 \pi}\right)^{s} \frac{1}{s} \\
& \quad-\frac{1}{2 \pi i}\left\{\int_{a+i U}^{b_{1}+i U}+\int_{b_{1}+i U}^{b_{1}-i U}+\int_{b_{1}-i U}^{a-i U}\right\} \frac{L^{\prime}}{L}(s, \chi \bar{\psi}) L^{2}\left(s, \omega_{0}^{\prime}\right)\left(\frac{T Q}{2 \pi}\right)^{s} \frac{d s}{s} \\
& \quad+O\left(\frac{T Q \log ^{2}(T Q)}{U}\right) .
\end{aligned}
$$

Note that

$$
\begin{aligned}
\operatorname{Res}_{s=1} & \frac{L^{\prime}}{L}(s, \chi \bar{\psi}) L^{2}\left(s, \omega_{0}^{\prime}\right)\left(\frac{T Q}{2 \pi}\right)^{s} \frac{1}{s} \\
& =\lim _{s \rightarrow 1} \frac{d}{d s}\left((s-1)^{2} \frac{L^{\prime}}{L}(s, \chi \bar{\psi}) L^{2}\left(s, \omega_{0}^{\prime}\right)\left(\frac{T Q}{2 \pi}\right)^{s} \frac{1}{s}\right) .
\end{aligned}
$$


To calculate this residue we use the following expansion (see [6, Sec. 4, formula (6); 20 , formula $(2.1 .16)]$ )

$$
\begin{aligned}
L^{2}\left(s, \omega_{0}^{\prime}\right) & =\left(\zeta(s) \prod_{p \mid Q}\left(1-p^{-s}\right)\right)^{2} \\
& =\left(\frac{1}{s-1}+\gamma+\sum_{n=1}^{\infty} \gamma_{n}(s-1)^{n}\right)^{2} \prod_{p \mid Q}\left(1-p^{-s}\right)^{2} \\
& =\left(\frac{1}{(s-1)^{2}}+\frac{2 \gamma}{(s-1)}+\sum_{m=0}^{\infty} a_{m}(s-1)^{m}\right) \prod_{p \mid Q}\left(1-p^{-s}\right)^{2},
\end{aligned}
$$

where $\gamma$ is the Euler constant, $\gamma_{n}, n=1,2, \ldots$, and $a_{m}, m=0,1, \ldots$ are absolute real constants. Then by (see $[1$, Theorem 2.4])

$$
\begin{aligned}
\prod_{p \mid Q}\left(1-p^{-1}\right) & =\frac{\phi(Q)}{Q}, \\
\frac{d}{d s}\left(\prod_{p \mid Q}\left(1-p^{-s}\right)\right)^{2} & =2 \prod_{p \mid Q}\left(1-p^{-s}\right)^{2} \sum_{p \mid Q} \frac{\log p}{p^{s}-1},
\end{aligned}
$$

and

$$
\frac{d}{d s} \frac{L^{\prime}}{L}(s, \chi \bar{\psi})=\left(\frac{L^{\prime \prime} L-\left(L^{\prime}\right)^{2}}{L^{2}}\right)(s, \chi \bar{\psi})
$$

we get

$$
\begin{aligned}
\operatorname{Res}_{s=1} & \frac{L^{\prime}}{L}(s, \chi \bar{\psi}) L^{2}\left(s, \omega_{0}^{\prime}\right)\left(\frac{T Q}{2 \pi}\right)^{s} \frac{1}{s} \\
= & \lim _{s \rightarrow 1} \frac{d}{d s}\left((1+2 \gamma(s-1)) \frac{L^{\prime}}{L}(s, \chi \bar{\psi})\left(\frac{T Q}{2 \pi}\right)^{s} \frac{1}{s} \prod_{p \mid Q}\left(1-p^{-s}\right)^{2}\right) \\
= & (2 \gamma-1) \frac{L^{\prime}}{L}(1, \chi \bar{\psi}) \frac{T Q}{2 \pi} \prod_{p \mid Q}\left(1-p^{-1}\right)^{2}+\frac{L^{\prime \prime} L-\left(L^{\prime}\right)^{2}}{L^{2}}(1, \chi \bar{\psi}) \frac{T Q}{2 \pi} \prod_{p \mid Q}\left(1-p^{-1}\right)^{2} \\
& +\prod_{p \mid Q}\left(1-p^{-1}\right)^{2} \frac{L^{\prime}}{L}(1, \chi \bar{\psi}) \frac{T Q}{2 \pi} \log \frac{T Q}{2 \pi}+2 \frac{L^{\prime}}{L}(1, \chi \bar{\psi}) \frac{T Q}{2 \pi} \\
& \times \prod_{p \mid Q}\left(1-p^{-1}\right)^{2} \sum_{p \mid Q} \frac{\log p}{p-1} \\
= & \left(\frac{\phi(Q)}{Q}\right)^{2} \frac{L^{\prime}}{L}(1, \chi \bar{\psi}) \frac{T Q}{2 \pi}\left(\log \frac{T Q}{2 \pi e}+2 \gamma+2 \sum_{p \mid Q} \frac{\log p}{p-1}+\left(\frac{L^{\prime \prime}}{L^{\prime}}-\frac{L^{\prime}}{L}\right)(1, \chi \bar{\psi})\right) .
\end{aligned}
$$


This and formulae (2.19), (2.18) yield

$$
\begin{aligned}
S_{1}= & -\psi(-1) \tau(\psi) \frac{\phi(Q)}{Q} \frac{L^{\prime}}{L}(1, \chi \bar{\psi}) \frac{T}{2 \pi}\left(\log \frac{T Q}{2 \pi e}+2 \gamma+2 \sum_{p \mid Q} \frac{\log p}{p-1}\right. \\
& \left.+\left(\frac{L^{\prime \prime}}{L^{\prime}}-\frac{L^{\prime}}{L}\right)(1, \chi \bar{\psi})\right)+\mathcal{E},
\end{aligned}
$$

where

$$
\begin{aligned}
\mathcal{E}= & -\frac{\psi(-1) \tau(\psi)}{2 \pi i \phi(Q)}\left\{\int_{a+i U}^{b_{1}+i U}+\int_{b_{1}+i U}^{b_{1}-i U}+\int_{b_{1}-i U}^{a-i U}\right\} \frac{L^{\prime}}{L}(s, \chi \bar{\psi}) \\
& \times L\left(s, \omega_{0}^{\prime}\right) L\left(s, \omega_{0}^{\prime \prime}\right)\left(\frac{T Q}{2 \pi}\right)^{s} \frac{d s}{s}+O\left(\frac{|\tau(\psi)|}{\phi(Q)} \frac{T Q \log ^{2}(T Q)}{U}\right) .
\end{aligned}
$$

Next we evaluate the integrals in $\mathcal{E}$. In view of inequality $\phi(Q) \gg Q^{1-\varepsilon}$ we obtain

$$
\frac{|\tau(\psi)|}{\phi(Q)} \ll Q^{-\frac{1}{2}+\varepsilon} .
$$

By this and by formulae (2.2), (2.13) we have that the first and the third integrals in $\mathcal{E}$ are bounded by

$$
\ll Q^{-\frac{1}{2}+\varepsilon}(\log ([q, Q] U))^{2}(Q U)^{\frac{3}{8}+\varepsilon} T Q / U .
$$

Next we consider the second integral in $\mathcal{E}$. We split it into three parts

$$
-\frac{1}{2 \pi i}\left\{\int_{b_{1}-i U}^{b_{1}-i}+\int_{b_{1}-i}^{b_{1}+i}+\int_{b_{1}+i}^{b_{1}+i U}\right\} \frac{L^{\prime}}{L}(s, \chi \bar{\psi}) L\left(s, \omega_{0}^{\prime}\right) L\left(s, \omega_{0}^{\prime \prime}\right)\left(\frac{T Q}{2 \pi}\right)^{s} \frac{d s}{s} .
$$

Again, using formulae (2.2) and (2.13) we see that the first and the third integrals in the last formula are bounded by

$$
\ll Q^{-\frac{1}{2}+\varepsilon}(\log ([q, Q] U))^{2}(Q U)^{\frac{3}{8}+\varepsilon}(T Q)^{b_{1}}
$$

and the second integral is bounded by

$$
\ll Q^{-\frac{1}{2}+\varepsilon}(T Q)^{b_{1}}(\log ([q, Q]))^{2} Q^{\frac{3}{8}+\varepsilon} .
$$

Now we choose $U=T^{1-b_{1}}$. By $Q \ll \log ^{A} T$ and $q \ll \log ^{B} T$ we obtain that

$$
\mathcal{E} \ll T^{1-\frac{c}{\log ^{\frac{3}{4}+\varepsilon} T}} .
$$

In the same way we get

$$
T_{1}=\delta(q, Q) \chi(-1) \tau\left(\chi \psi_{0}\right) \frac{1}{\phi(Q)} \frac{T Q}{2 \pi} L^{2}(1, \chi \bar{\psi})+O\left(T^{1-\frac{c}{\log ^{\frac{3}{4}+\varepsilon} T}}\right)
$$


and

$$
T_{2} \ll T^{1-\frac{c}{\log ^{\frac{3}{4}+\varepsilon} T}}
$$

Under GRH we choose $b_{1}=1 / 2+1 / \log (Q T), U=Q T$ and make use of the bound (2.4). This finishes the proof of Lemma 2.2 .

Lemma 2.3. Let $\psi \bmod Q$ be a primitive Dirichlet character. Then, for $x \rightarrow \infty$,

$$
\begin{aligned}
& \sum_{m n \leq x} \psi(m) \psi(n) \exp \left(-2 \pi i \frac{m n}{Q}\right) \\
& \quad=-\psi(-1) \tau(\psi) \frac{\phi(Q)}{Q^{2}} x\left(\log \frac{x}{e}+2 \gamma+2 \sum_{p \mid Q} \frac{\log p}{p-1}\right)+O\left(Q^{-\frac{1}{8}+\varepsilon} x^{\frac{11}{16}+\varepsilon}+Q^{4}\right)
\end{aligned}
$$

uniformly in $Q$. Under GRH the error term can be replaced by $O\left(Q^{-1 / 2+\varepsilon} x^{1 / 2+\varepsilon}+\right.$ $\left.Q^{4}\right)$ uniformly in $Q$.

Proof. By the orthogonality of Dirichlet characters the sum in Lemma 2.3 can be written as

$$
\begin{aligned}
S & =\sum_{m n \leq x} \psi(m) \psi(n) \exp \left(-2 \pi i \frac{m n}{Q}\right) \\
& =\frac{1}{\phi^{2}(Q)} \sum_{\eta \bmod } \sum_{\omega \bmod } \sum_{Q=1}^{Q} \bar{\eta}(a) \psi(a) \sum_{b=1}^{Q} \bar{\omega}(b) \psi(b) \exp \left(-2 \pi i \frac{a b}{Q}\right) \sum_{m n \leq x} \eta(m) \omega(n) \\
& =\psi(-1) \tau(\psi) \frac{1}{\phi(Q)} \sum_{m n \leq x} \eta_{0}(m) \omega_{0}(n)+O\left(\phi^{4}(Q)\right) .
\end{aligned}
$$

Perron's formula yields

$$
\sum_{m n \leq x} \eta_{0}(n) \omega_{0}(l)=-\frac{1}{2 \pi i} \int_{a-i U}^{a+i U} L\left(s, \eta_{0}\right) L\left(s, \omega_{0}\right) x^{s} \frac{d s}{s}+O\left(\frac{x \log ^{2} x}{U}\right),
$$

where $a=1 / \log x$. Let $b_{1}=1 / 2+1 / \log x$. Shifting the line of integration we get

$$
\begin{aligned}
& \sum_{m n \leq x} \eta_{0}(n) \omega_{0}(l) \\
& =-\operatorname{Res}_{s=1} L\left(s, \eta_{0}\right) L\left(s, \omega_{0}\right) x^{s} \frac{1}{s} \\
& \quad+\frac{1}{2 \pi i}\left\{\int_{a+i U}^{b_{1}+i U}+\int_{b_{1}+i U}^{b_{1}-i U}+\int_{b_{1}-i U}^{a-i U}\right\} L\left(s, \eta_{0}\right) L\left(s, \omega_{0}\right) x^{s} \frac{d s}{s} \\
& \quad+O\left(\frac{x \log ^{2} x}{U}\right),
\end{aligned}
$$


The definition of residue, formulae (2.20) and (2.21) lead to

$$
\operatorname{Res}_{s=1} L\left(s, \eta_{0}\right) L\left(s, \omega_{0}\right) x^{s} \frac{1}{s}=\frac{\phi^{2}(Q)}{Q^{2}} x\left(\log \frac{x}{e}+2 \gamma+2 \sum_{p \mid Q} \frac{\log p}{p-1}\right) .
$$

Hence, we have

$$
S=-\psi(-1) \tau(\psi) \frac{\phi(Q)}{Q^{2}} x\left(\log (x / e)+2\left(\gamma+\sum_{p \mid Q} \frac{\log p}{p-1}\right)\right)+O\left(\phi^{4}(Q)\right)+\mathcal{E},
$$

where

$$
\begin{aligned}
\mathcal{E}= & \psi(-1) \tau(\psi) \frac{1}{2 \pi i \phi(Q)}\left\{\int_{a+i U}^{b_{1}+i U}+\int_{b_{1}+i U}^{b_{1}-i U}+\int_{b_{1}-i U}^{a-i U}\right\} L\left(s, \eta_{0}\right) L\left(s, \omega_{0}\right) x^{s} \frac{d s}{s} \\
& +O\left(\frac{|\tau(\psi)|}{\phi(Q)} \frac{x \log ^{2} x}{U}\right) .
\end{aligned}
$$

It remains to bound $\mathcal{E}$. By formulae $(2.2)$ and $(2.22)$ we have that the first and the last integrals in $\mathcal{E}$ are bounded by $\ll Q^{-1 / 2+\varepsilon}(Q U)^{3 / 8+\varepsilon} x / U$. The second integral in $\mathcal{E}$ we split into three parts

$$
\left\{\int_{b_{1}-i U}^{b_{1}-i}+\int_{b_{1}-i}^{b_{1}+i}+\int_{b_{1}+i}^{b_{1}+i U}\right\} L\left(s, \eta_{0}\right) L\left(s, \omega_{0}\right) x^{s} \frac{d s}{s} .
$$

In the last formula the first and the third integrals are both bounded by $\ll$ $Q^{-1 / 2+\varepsilon}(Q U)^{3 / 8+\varepsilon} x^{1 / 2}$ and the second integral is bounded by $\ll Q^{-1 / 2+\varepsilon} x^{1 / 2}$. We choose $U=x^{1 / 2}$. Then

$$
\mathcal{E} \ll Q^{-\frac{1}{8}+\varepsilon} x^{\frac{11}{16}+\varepsilon} .
$$

If we assume GRH then the bound $(2.4)$ gives $\mathcal{E} \ll Q^{-1 / 2+\varepsilon} x^{1 / 2+\varepsilon}$. Lemma 2.3 is proved.

\section{Proof of Theorem 1.1}

Let $\chi \bmod q$ and $\psi \bmod Q$ be primitive Dirichlet characters such that $\chi \neq \psi$. The proof of the theorem relies on the method of [4]. The idea is to interpret the sum in question as a sum of residues, respectively a contour integral

$$
\sum_{0<\gamma_{\chi} \leq T} L\left(\rho_{\chi}, \psi\right) L\left(1-\rho_{\chi}, \bar{\psi}\right)=\frac{1}{2 \pi i} \int_{\mathfrak{C}} \frac{L^{\prime}}{L}(s, \chi) L(s, \psi) L(1-s, \bar{\psi}) d s,
$$

which can be evaluated by the modified Gonek lemma (Lemma 2.1). We choose an appropriate path of integration $\mathfrak{C}$. In view of the bound for zeros (2.11) we can choose $\frac{1}{\log q} \ll b \leq 1$ and $T \geq 2$ such that

$$
\min _{\gamma_{\chi}}\left|b-\gamma_{\chi}\right| \gg \frac{1}{\log q} \text { and } \min _{\gamma_{\chi}}\left|T-\gamma_{\chi}\right| \gg \frac{1}{\log (q T)} .
$$


Let $a=1+1 / \log (Q T)$ and define the contour $\mathfrak{C}$ to be the rectangle with vertices $a+i b, a+i T, 1-a+i T, 1-a+i b$. Then

$$
\begin{aligned}
& \frac{1}{2 \pi i} \int_{\mathfrak{C}} \frac{L^{\prime}}{L}(s, \chi) L(s, \psi) L(1-s, \bar{\psi}) d s \\
& \quad=\frac{1}{2 \pi i}\left\{\int_{a+i b}^{a+i T}+\int_{a+i T}^{1-a+i T}+\int_{1-a+i T}^{1-a+i b}+\int_{1-a+i b}^{a+i b}\right\} \frac{L^{\prime}}{L}(s, \chi) L(s, \psi) L(1-s, \bar{\psi}) d s \\
& \quad=: \sum_{j=1}^{4} \mathcal{J}_{j} .
\end{aligned}
$$

First we consider

$$
\mathcal{J}_{1}=\frac{1}{2 \pi i} \int_{a+i}^{a+i T} \frac{L^{\prime}}{L}(s, \chi) L(s, \psi) L(1-s, \bar{\psi}) d s+O(1) .
$$

By applying the functional equation (2.1) we get

$$
\begin{aligned}
\mathcal{J}_{1}= & \tau(\bar{\psi}) \frac{1}{Q} \frac{1}{2 \pi i} \int_{a+i b}^{a+i T}\left(\frac{Q}{2 \pi}\right)^{s} \Gamma(s) \exp \left(-\frac{\pi i s}{2}\right) \frac{L^{\prime}}{L}(s, \chi) L^{2}(s, \psi) d s \\
& +\tau(\bar{\psi}) \psi(-1) \frac{1}{Q} \frac{1}{2 \pi i} \int_{a+i b}^{a+i T}\left(\frac{Q}{2 \pi}\right)^{s} \Gamma(s) \exp \left(\frac{\pi i s}{2}\right) \frac{L^{\prime}}{L}(s, \chi) L^{2}(s, \psi) d s \\
= & \mathcal{J}_{11}+\mathcal{J}_{12} .
\end{aligned}
$$

In view of the modified Gonek lemma (Lemma 2.1) we have

$$
\mathcal{J}_{11}=-\tau(\bar{\psi}) \frac{1}{Q} \sum_{m n l \leq \frac{T Q}{2 \pi}} \Lambda(m) \chi(m) \psi(n) \psi(l) \exp \left(-2 \pi i \frac{m n l}{Q}\right)+O\left((Q T)^{\frac{1}{2}+\varepsilon}\right)
$$

and $\mathcal{J}_{12}=O\left(Q^{\frac{1}{2}}\right)$. By this, Lemma 2.2, and the equality

$$
\tau(\bar{\psi}) \psi(-1) \tau(\psi)=Q
$$

we obtain

$$
\begin{aligned}
\mathcal{J}_{1}= & \frac{\phi(Q)}{Q} \frac{L^{\prime}}{L}(1, \chi \bar{\psi}) \frac{T}{2 \pi}\left(\log \frac{T Q}{2 \pi e}+2 \gamma+2 \sum_{p \mid Q} \frac{\log p}{p-1}+\left(\frac{L^{\prime \prime}}{L^{\prime}}-\frac{L^{\prime}}{L}\right)(1, \chi \bar{\psi})\right) \\
& -\delta(q, Q)\left(\chi \psi_{0}\right)(-1) \tau\left(\chi \psi_{0}\right) \frac{\tau(\bar{\psi})}{\phi(Q)} \frac{T}{2 \pi} L^{2}(1, \chi \bar{\psi})+O\left(T^{1-\frac{c}{\log ^{\frac{3}{4}+\varepsilon} T}}\right) .
\end{aligned}
$$

Under GRH the error term in (3.5) can be replaced by $O\left(q^{1+\varepsilon} Q^{\varepsilon} T^{\frac{1}{2}+\varepsilon}+\right.$ $\left.q Q^{9 / 2+\varepsilon} T^{\varepsilon}+(Q T)^{1 / 2+\varepsilon}\right)$.

Second we consider $\mathcal{J}_{2}$ and $\mathcal{J}_{4}$. Using bounds (2.2), (2.3), and (2.13) we get

$$
\begin{aligned}
\mathcal{J}_{2} & =\frac{1}{2 \pi i} \int_{a+i T}^{1-a+i T} \frac{L^{\prime}}{L}(s, \chi) L(s, \psi) L(1-s, \bar{\psi}) d s \\
& =O\left((Q T)^{11 / 16+\varepsilon} \log ^{2}(q T)\right) .
\end{aligned}
$$


Similarly,

$$
\mathcal{J}_{4} \ll(Q)^{11 / 16+\varepsilon} \log ^{2} q .
$$

Under GRH we use (2.4) instead of $(2.2)$ and we obtain $\mathcal{J}_{2}, \mathcal{J}_{4} \ll(Q T)^{1 / 2+\varepsilon}$ $\log ^{2}(q T)$.

Next we consider $\mathcal{J}_{3}$. A change of variables $s \mapsto 1-\bar{s}$ gives

$$
\mathcal{J}_{3}=-\frac{1}{2 \pi i} \int_{a+i b}^{a+i T} \frac{L^{\prime}}{L}(1-\bar{s}, \chi) L(1-\bar{s}, \psi) L(\bar{s}, \bar{\psi}) d s .
$$

By complex conjugation we get

$$
\overline{\mathcal{J}_{3}}=-\frac{1}{2 \pi i} \int_{a+i b}^{a+i T} \frac{L^{\prime}}{L}(1-s, \bar{\chi}) L(1-s, \bar{\psi}) L(s, \psi) d s .
$$

The functional equation (2.1) and its logarithmic derivative (2.9) together with the property (2.8) lead to

$$
\begin{aligned}
\frac{L^{\prime}}{L}(1 & -s, \bar{\chi}) L(1-s, \bar{\psi}) \\
= & \left(\frac{\Delta^{\prime}}{\Delta}(s, \chi)-\frac{L^{\prime}}{L}(s, \chi)\right) \\
& \times \tau(\bar{\psi}) \frac{1}{Q}\left(\frac{Q}{2 \pi}\right)^{s} \Gamma(s)\left(\exp \left(-\frac{\pi i s}{2}\right)+\psi(-1) \exp \left(+\frac{\pi i s}{2}\right)\right) L(s, \psi) .
\end{aligned}
$$

Then

$$
\begin{aligned}
\overline{\mathcal{J}_{3}}= & -\tau(\bar{\psi}) \frac{1}{2 \pi i Q} \int_{a+i b}^{a+i T} \frac{\Delta^{\prime}}{\Delta}(s, \chi)\left(\frac{Q}{2 \pi}\right)^{s} \Gamma(s) \exp \left(-\frac{\pi i s}{2}\right) L^{2}(s, \psi) d s \\
& -\psi(-1) \tau(\bar{\psi}) \frac{1}{2 \pi i Q} \int_{a+i b}^{a+i T} \frac{\Delta^{\prime}}{\Delta}(s, \chi)\left(\frac{Q}{2 \pi}\right)^{s} \Gamma(s) \exp \left(\frac{\pi i s}{2}\right) L^{2}(s, \psi) d s \\
& +\tau(\bar{\psi}) \frac{1}{2 \pi i Q} \int_{a+i b}^{a+i T}\left(\frac{Q}{2 \pi}\right)^{s} \Gamma(s) \exp \left(-\frac{\pi i s}{2}\right) \frac{L^{\prime}}{L}(s, \chi) L^{2}(s, \psi) d s \\
& +\psi(-1) \tau(\bar{\psi}) \frac{1}{2 \pi i Q} \int_{a+i b}^{a+i T}\left(\frac{Q}{2 \pi}\right)^{s} \Gamma(s) \exp \left(\frac{\pi i s}{2}\right) \frac{L^{\prime}}{L}(s, \chi) L^{2}(s, \psi) d s \\
= & \sum_{j=1}^{4} \mathcal{F}_{j},
\end{aligned}
$$

say.

First we consider $\mathcal{F}_{1}$. We rewrite $\mathcal{F}_{1}$ in the following way:

$$
\begin{aligned}
\mathcal{F}_{1}= & -\frac{1}{Q} \tau(\bar{\psi}) \int_{b}^{T} \frac{\Delta^{\prime}}{\Delta}(a+i \tau, \chi) d \\
& \times\left(\frac{1}{2 \pi i} \int_{a+i}^{a+i \tau}\left(\frac{Q}{2 \pi}\right)^{s} \Gamma(s) \exp \left(-\frac{\pi i s}{2}\right) L^{2}(s, \psi) d s\right) .
\end{aligned}
$$


By Lemmas 2.1 and 2.3 we get

$$
\begin{aligned}
& \frac{1}{2 \pi i} \int_{a+i}^{a+i \tau}\left(\frac{Q}{2 \pi}\right)^{s} \Gamma(s) \exp \left(-\frac{\pi i s}{2}\right) L^{2}(s, \psi) d s \\
& \quad=\sum_{m n \leq \frac{\tau Q}{2 \pi}} \psi(m) \psi(n) \exp \left(-2 \pi i \frac{m n}{Q}\right)+O\left((Q \tau)^{\frac{1}{2}+\varepsilon}\right) \\
& \quad=\psi(-1) \tau(\psi) \frac{\phi(Q)}{Q} \frac{\tau}{2 \pi}\left(\log \frac{\tau Q}{2 \pi e}+2 \gamma+2 \sum_{p \mid Q} \frac{\log p}{p-1}\right)+O\left(Q^{-\frac{1}{8}+\varepsilon} \tau^{\frac{11}{16}+\varepsilon}+Q^{4}\right) .
\end{aligned}
$$

Hence, in view of the asymptotic formula (2.8) for the logarithmic derivative of the delta function and the property (3.4) of the Gauss sum we obtain

$$
\begin{aligned}
\mathcal{F}_{1}= & -\frac{\phi(Q)}{Q} \int_{b}^{T}\left(-\log \frac{\tau q}{2 \pi}+O\left(\frac{1}{\tau}\right)\right) \\
& \times d\left(\frac{\tau}{2 \pi}\left(\log \frac{\tau Q}{2 \pi e}+2 \gamma+2 \sum_{p \mid Q} \frac{\log p}{p-1}\right)+O\left(Q^{-\frac{1}{8}+\varepsilon} \tau^{\frac{11}{16}+\varepsilon}+Q^{4}\right)\right) \\
= & \frac{\phi(Q)}{Q} \frac{T}{2 \pi}\left(\log \frac{T q}{2 \pi e} \log \frac{T Q}{2 \pi e}+2 \log \frac{T q}{2 \pi e}\left(\gamma+\sum_{p \mid Q} \frac{\log p}{p-1}\right)+1\right) \\
& +O\left(Q^{-\frac{1}{8}+\varepsilon} T^{\frac{11}{16}+\varepsilon} \log q+Q^{4} \log (q T)\right) .
\end{aligned}
$$

Under GRH the error term for $\mathcal{F}_{1}$ can be replaced by $O\left(Q^{-1 / 2+\varepsilon} T^{1 / 2+\varepsilon} \log q+\right.$ $\left.Q^{4} \log (q T)\right)$.

Reasoning similarly to $\mathcal{F}_{1}$ we obtain

$$
\begin{aligned}
\mathcal{F}_{2}= & -\frac{1}{Q} \psi(-1) \tau(\bar{\psi}) \int_{b}^{T} \frac{\Delta^{\prime}}{\Delta}(a+i \tau, \chi) \\
& \times d\left(\frac{1}{2 \pi i} \int_{a+i}^{a+i \tau}\left(\frac{Q}{2 \pi}\right)^{s} \Gamma(s) \exp \left(\frac{\pi i s}{2}\right) L(s, \psi) d s\right) \\
= & O\left(Q^{\frac{1}{2}} \log (q T)\right) .
\end{aligned}
$$

Under GRH for $\mathcal{F}_{2}$ we use the same bound as in (3.10).

We turn to the integral $\mathcal{F}_{3}$. Using Lemmas 2.1 and 2.2 we get

$$
\begin{aligned}
\mathcal{F}_{3}= & -\frac{1}{Q} \tau(\bar{\psi}) \sum_{m n l \leq \frac{T Q}{2 \pi}} \Lambda(m) \chi(m) \psi(n) \psi(l) \exp \left(-2 \pi i \frac{m n l}{Q}\right)+O\left(Q^{\frac{1}{2}} T^{\frac{1}{2}+\varepsilon}\right) \\
= & \frac{\phi(Q)}{Q} \frac{L^{\prime}}{L}(1, \chi \bar{\psi}) \frac{T}{2 \pi}\left(\log \frac{T Q}{2 \pi e}+2 \gamma+2 \sum_{p \mid Q} \frac{\log p}{p-1}+\left(\frac{L^{\prime \prime}}{L^{\prime}}-\frac{L^{\prime}}{L}\right)(1, \chi \bar{\psi})\right) \\
& -\delta(q, Q)\left(\chi \psi_{0}\right)(-1) \tau\left(\chi \psi_{0}\right) \frac{\tau(\bar{\psi})}{\phi(Q)} \frac{T}{2 \pi} L^{2}(1, \chi \bar{\psi})+O\left(T^{1-\frac{c}{\log ^{\frac{3}{4}+\varepsilon} T}}\right) .
\end{aligned}
$$


Under GRH the error term can be replaced by $O\left(q^{1+\varepsilon} Q^{\varepsilon} T^{\frac{1}{2}+\varepsilon}+q Q^{9 / 2+\varepsilon} T^{\varepsilon}+\right.$ $\left.(Q T)^{1 / 2+\varepsilon}\right)$.

In a similar way as above we have

$$
\mathcal{F}_{4} \ll Q^{\frac{1}{2}} \log (q T) .
$$

The last bound is used also under GRH.

Now in view of (3.1), (3.3), (3.5)-(3.12) we obtain main asymptotic formula (1.1). Accordingly, the notes after each formula (3.1), (3.3), (3.5)-(3.12) give the error term under GRH. By this Theorem 1.1 is proved.

\section{Proof of Corollary 1.4}

Let

$$
S_{1}:=\sum_{0<\gamma_{\chi} \leq T} L\left(\rho_{\chi}, \psi\right) \text { and } S_{2}:=\sum_{0<\gamma_{\chi} \leq T}\left|L\left(\rho_{\chi}, \psi\right)\right|^{2} .
$$

By Hölder's inequality we get

$$
\sum_{\substack{0<\gamma_{\chi} \leq T \\ L\left(1 / 2+\gamma_{\chi}, \psi\right) \neq 0}} 1 \geq \frac{\left|S_{1}\right|^{2}}{S_{2}}
$$

To evaluate sums $S_{1}$ and $S_{2}$ we will use Theorems 1.3 and 1.1. First we state several helpful inequalities. From [6, Sec. 14, formulae (11) and (13)], for a nonprincipal Dirichlet character $\chi \bmod q$, we have

$$
|L(1, \chi)| \ll \log q, \quad\left|L^{\prime}(1, \chi)\right| \ll \log ^{2} q, \quad\left|L^{\prime \prime}(1, \chi)\right| \ll \log ^{3} q .
$$

By Siegel's theorem (see [6, Sec. 21]), for a real primitive Dirichlet character $\chi \bmod$ $q$, we have

$$
|L(1, \chi)| \gg q^{-\varepsilon}
$$

If $\chi \bmod q$ is a complex primitive character we use the zero free region of $L(s, \chi)$ (see [6, Sec. 14, formula (6)]) and follow the proof of [15, Corollary 7] to obtain the lower bound

$$
|L(1, \chi)| \gg q^{-\varepsilon} \text {. }
$$

We will show that the lower bounds (4.2) and (4.3) are valid also for an imprimitive character $\chi$. Indeed, if $\chi$ is the imprimitive character induced by a primitive character $\chi_{1}$ then

$$
L(s, \chi)=L\left(s, \chi_{1}\right) \prod_{p \mid q}\left(1-\frac{\chi_{1}(p)}{p^{s}}\right) .
$$

Thus for imprimitive character the bounds (4.2) and (4.3) follow by

$$
\left|\prod_{p \mid q}\left(1-\frac{\chi_{1}(p)}{p}\right)\right| \geq \prod_{p \mid q}\left(1-\frac{1}{p}\right)=\frac{\phi(q)}{q} \gg q^{-\varepsilon} .
$$


By above we have that

$$
\delta(q, Q) L(1, \chi \bar{\psi}) \psi(-1) \tau(\psi) \frac{\tau\left(\bar{\chi} \psi_{0}\right)}{\phi(Q)} \frac{T}{2 \pi} \ll T Q^{\frac{1}{2}} \log Q \ll T \log ^{1-\varepsilon} T
$$

and

$$
\frac{L^{\prime}}{L}(1, \psi \bar{\chi}) \frac{T}{2 \pi} \ll(q Q)^{\varepsilon} T \ll T \log ^{\varepsilon} T .
$$

This and Theorem 1.3 give that

$$
S_{1} \gg T \log T \text {. }
$$

We will find an upper bound for $\left|S_{2}\right|$. We have

$$
\begin{gathered}
\sum_{p \mid Q} \frac{\log p}{p-1} \leq \log Q \sum_{p \leq Q} \frac{1}{p-1} \ll \log ^{\epsilon} T, \\
\delta(q, Q)\left(\tau\left(\chi \psi_{0}\right) \tau(\bar{\psi}) L^{2}(1, \chi \bar{\psi})+\tau\left(\bar{\chi} \psi_{0}\right) \tau(\psi) L^{2}(1, \bar{\chi} \psi)\right) \frac{\left(\chi \psi_{0}\right)(-1)}{\phi(Q)} \frac{T}{2 \pi} \\
\ll Q^{\frac{1}{2}} T \log ^{2} Q \ll T \log T,
\end{gathered}
$$

and

$$
\frac{L^{\prime \prime}}{L}(1, \psi \bar{\chi}) \frac{T}{2 \pi} \ll(q Q)^{\varepsilon} T \ll T \log T .
$$

Then Theorem 1.1 leads to

$$
S_{2} \ll \frac{\phi(Q)}{Q} T \log ^{2} T .
$$

Now the corollary follows by formula (4.1).

\section{Acknowledgments}

The authors are supported by grant No. MIP-066/2012 from the Research Council of Lithuania. The second author is grateful to his friend Gintautas Aidietis for support.

\section{References}

[1] T. M. Apostol, Introduction to Analytic Number Theory, 5th edn. (Springer, New York, 1998).

[2] J. B. Conrey, A. Ghosh and S. M. Gonek, Simple zeros of the zeta function of a quadratic number field, I, Invent. Math. 86 (1986) 563-576.

[3] Simple zeros of the zeta function of a quadratic number field, II, in Analytic Number Theory and Diophantine Problems, eds. A. C. Adolphson et al. (Birkhäuser, 1987), pp. 87-114.

[4] Simple zeros of zeta functions, Publ. Math. Orsay 88(02) (1988) 77-83.

[5] Simple zeros of the Riemann zeta-function, Proc. London Math. Soc. 76(3) (1998) 497-522. 
[6] H. Davenport, Multiplicative Number Theory, 2nd edn., Revised by H. L. Montgomery (Springer-Verlag, 1980).

[7] A. Fujii, On the zeros of Dirichlet L-functions. V, Acta Arith. 28 (1976) 395-403.

[8] - Some Observations concerning the distribution of the zeros of the zeta functions (II), Comment. Math. Univ. St. Pauli 40 (1991) 125-231.

[9] Explicit formulas and oscillations, in Emerging Applications of Number Theory, IMA Volumes in Mathematics and its Applications, Vol. 109 (Springer, 1999), pp. 219-267.

[10] R. Garunkštis, On the Backlund equivalent for the Lindelöf hypothesis, Adv. Stud. Pure Math. 49 (2007) 91-104.

[11] R. Garunkštis, J. Kalpokas and J. Steuding, Sum of the Dirichlet L-Functions over nontrivial zeros of another Dirichlet L-Function, Acta Math. Hungar. 128(3) (2010) $287-298$.

[12] S. M. Gonek, Mean values of the Riemann zeta-function and its derivatives, Invent. Math. 75 (1984) 123-141.

[13] D. R. Heath-Brown, Hybrid bounds for Dirichlet $L$-functions II, Q. J. Math. 31 (1980) 157-167.

[14] D. A. Kaptan, Y. Karabulut and C. Y. Yildirim, Some mean value theorems for the Riemann zeta-function and Dirichlet L-functions, Comment. Math. Univ. St. Pauli 60(1-2) (2011) 83-87.

[15] X. Li, Upper bounds on $L$-functions at the edge of the critical strip, Int. Math. Res. Not. 2010(4) (2010) 727-755.

[16] A. Perelli, A survey of the Selberg class of L-functions, Part II, Riv. Mat. Univ. Parma (7) 3* (2004) 83-118.

[17] K. Prachar, Primzahlverteilung (Springer-Verlag, Wien, 1957).

[18] R. Raghunathan, A comparison of zeros of L-functions, Math. Res. Lett. 6(2) (1999) $155-167$.

[19] J. Steuding, Dirichlet series associated to periodic arithmetic functions and the zeros of Dirichlet $L$-functions, in Analytic and Probabilistic Methods Number Theory (TEV, Vilnius, 2002), pp. 282-296.

[20] E. C. Titchmarsh, The Theory of the Riemann Zeta-Function, 2nd edn., Revised by D. R. Heath-Brown (Oxford University Press, 1986). 